\title{
Analysis of Injuries and Risk Factors in Taekwondo during the 2014 Italian University Championship
}

\author{
Mattia Fortina $^{1}$ Simone Mangano ${ }^{2}$ Serafino Carta ${ }^{1}$ Christian Carulli ${ }^{3}$ \\ ${ }^{1}$ University Department of Orthopaedic and Traumatology, Hospital \\ Address for correspondence Mattia Fortina, MD, University \\ of Siena, Siena, Italy \\ 2 Department of Physical Medicine and Rehabilitation, University \\ Hospital of Milan, Milan, Italy \\ Department of Orthopaedic and Traumatology, Hospital of Siena, \\ viale Mario Bracci n.16, Siena 53100, Italy \\ (e-mail: mattiafortina@me.com).
}

${ }^{3}$ Orthopaedic Clinic, University of Florence, Florence, Italy

Joints 2017;5:168-172.

\begin{abstract}
Purpose This study aims to analyze the risk factors and type of injuries occurring in Taekwondo athletes participating in a national competition.

Methods Out of the 127 competitors, 18 athletes got injured during a Taekwondo championship who were analyzed for the following parameters: modality of training; age, weight; belt color; and the type of injury.

Results Around $89 \%$ of the injuries were due to bruising and were found mainly on the lower limbs (61\%) during the elimination rounds and during the first match of the day. The higher probability of injury was in the second round (56\%), and during the first match of the day (72\%). Nearly all the athletes were able to complete the game in which they were injured (83\%). Comparing the average age of the athletes suffering an injury ( $23.6 \pm 2.06$ years) with their average years of training ( $8.4 \pm 7.05$ years) it can be noted that these athletes began this discipline rather late. The more is the training age

\section{Keywords}

- athletic injuries

- injury rate

- prevention

- risk factors

- martial arts

- Taekwondo and the weekly hours of training, the more are the numbers of matches completed, even as injured. Beginners with a low-level belt suffered more injuries than the experienced subjects did.

Conclusion The following risk factors for injury were found: starting to practice in late age, weekly training sessions with a few number of hours, male sex, low-level belt, elimination rounds, the first match of the day, and second round.

Level of Evidence Level III, observational analytic study without a control group.
\end{abstract}

\section{Introduction}

Practicing sports is strongly suggested to stay healthy and to prevent pathologies related to a sedentary lifestyle. Nonetheless, although the benefits of physical activity are evident, in some sports there is a high risk of injury. Identifying specific risk factors is important to prevent such risks. These factors can be either intrinsic or extrinsic; the former include specific characteristics of the individual athlete (age, sex, weight, and height), while the latter refers to the external environment that might influence the athlete (protective gear, competition rules). Thus, it is crucial to evaluate those risk factors that can be influenced or modified, such as weight, technical ability, and physical fitness. ${ }^{1}$

Taekwondo is one of the most practiced full-contact sports. It is estimated that there are approximately 80 million subjects practicing Taekwondo in all continents. The athletes are equipped with protective gear (head, mouth, forearm, and shin guards; padded trunk protector; gloves; groin pads; and footwear), and they face each other in an $8 \mathrm{~m} \times 8 \mathrm{~m}$ square. Valid hits to gain points must be directed exclusively at chest published online August 4, 2017
DOI https://doi.org/ $10.1055 / \mathrm{s}-0037-1605390$. ISSN 2282-4324.
Copyright @ 2017 Georg Thieme Verlag KG Stuttgart · New York
License terms

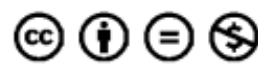


or face of the opponent using the feet; fists can only be directed at the chest to be valid.

The main cause of injury in Taekwondo derives from direct contact between the two athletes, and one of the major risks is an inefficient protection from a blow received by the competitor. ${ }^{2}$ Both competitions and training sessions are potentially at risk of injuries, which represent the main reason to interrupt a match or even cause hospitalization. ${ }^{3}$ Feet and head are the most involved site of injury. ${ }^{2-4}$

The level of competitions is related to specific differences in the type and frequency of injuries. Mild-to-severe injuries are common in a low-level of participation due to less experience and competence, while moderate-to-severe injuries may regard high-level subjects, due to more potent and fine fighting technique. ${ }^{5}$ Also, the use of protective gear usually seems to protect the one attacking rather than the one defending. ${ }^{6}$

Few articles ${ }^{7-9}$ have thoroughly analyzed the risk of injury mainly during Taekwondo competitions. The aim of this prospective study is the analysis of risk factors and type of injuries occurring in Taekwondo athletes participating in a national competition.

\section{Methods}

The National University Championship of Taekwondo was organized from May 17 to 18, 2014 at the "Pavesi Center" in Milan. Athletes of both sexes and any nationality could participate with specific inclusion criteria: born between January 1, 1986 and December 31, 1996; active membership of the Taekwondo Italian Federation (FITA); enrollment in a study course (Bachelor's degree, Honors Bachelor's degree, Postgraduate diploma, PhD, Master's degree) at an Italian university or Academies of Fine Arts and Music Conservatories, being in possession of a secondary school diploma.

The present study has been approved by the ethical committee of the University Hospital of Milan and was conducted attending the requirements of the Declaration of Helsinki. All athletes gave their informed consent to participate in the study before starting the competition.

A population of 127 athletes participating in the competition was divided according to their sex (93 males, 34 females), weight category, and belt color. Only the athletes that suffered at least one injury during the 2-day competition were included in the study. Age of start of training, the number of hours of training and competitions, weight, height, belt level, and other parameters were recorded for all athletes, as type and site of injury, the request of the medical team, drop off of the match, and other events related to the competition were noted.

Every injury was recorded, and in the case of multiple lesions, each was separately documented. Data were registered immediately after the medical staff intervention or at the end of the match on a specific questionnaire ( - Table $\mathbf{1}$ ) by one of the authors. In the martial art culture, the black belt represents maturity, a good level of skill, rejection of darkness and fear. These qualities are not present in the other belts that have more to learn about self-control. Hence, we decided to split the type of belt into only two groups (colored/black), as the World Taekwondo Federation makes for the competition rules in the junior division. The time phase in which the injury occurred (morning/afternoon), and the type of action performed (attack/defense) were also recorded. Moreover, three categories of injuries were planned regarding the region of interest: head (head and neck), upper limbs (hands, forearms, arms, shoulders, chest, stomach, and back), and lower limbs (feet, legs, thighs, and pelvis).

A statistical association between the quantitative variables was evaluated through the Pearsons' linear correlation coefficient correlation. Student's $t$-test was used for comparison between the two groups for the quantitative variable. The results were considered statistically significant when the $p$ value was less than 0.05 .

\section{Results}

A total of 18 athletes reported an injury among 127 participants to the competition (7.1\%). Out of these 18,14 were males (15.0\%) and 4 were females (11.8\%). The type of injuries and other data are shown in - Table 1.

Analyzing the whole sample group (-Table 2), two negative correlations were found. One between the duration of Taekwondo practice, expressed in years and referred as "training age," and the number of weekly training sessions $(r=-0.62 ; p=0.006)$, and one between the "training age" and the number of hours of training per week $(r=-0.55$; $p=0.017$ ).

Male athletes showed a statistically significant negative correlation between the "training age" and the number of matches completed $(r=-0.56 ; p=0.038)$. Athletes that were injured while defending showed a significant negative correlation between the "training age" and the number of matches completed ( $r=-0.69 ; p=0.013)$. Athletes ending their game even injured showed a significant correlation between height and the number of matches completed $(r=0.55 ; p=0.032)$. Worthy of note, the association was observed regardless of requested intervention of the medical team on-site. This group also obtained a marked result when comparing the number of hours of weekly training sessions and the number of matches completed ( $r=0.99 ; p=0.006$ ). Regarding the black belt athletes, a significant correlation was observed between height and the number of matches completed $(r=0.72 ; p=0.046)$. The Student's $t$-test showed a significant difference between types of the belt for the training age and the number of matches completed ( $p=0.001$ and 0.018 , respectively). Other results are summarized in - Table 3.

\section{Discussion}

Comparing the average age of the athletes suffering an injury $(23.6 \pm 2.06$ years $)$ with their average years of training ( $8.4 \pm 7.05$ years) it can be noted that these athletes began this discipline rather late. It has certainly had some influence regarding the learning technique of this sport, which 


\begin{tabular}{|c|c|c|c|c|c|c|c|c|c|c|c|c|c|c|c|c|c|c|c|c|c|}
\hline 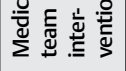 & 2 & 2 & 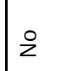 & 2 & 2 & $\frac{0}{2}$ & $\stackrel{\check{y}}{\searrow}$ & 2 & 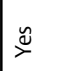 & 2 & 2 & 2 & 2 & $\stackrel{\check{\nu}}{\check{0}}$ & $\stackrel{\check{u}}{\rightleftharpoons}$ & z & 2 & $\frac{8}{2}$ & I & $\frac{0}{2}$ & $\stackrel{\infty}{\wedge}$ \\
\hline 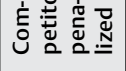 & $\frac{9}{2}$ & $\frac{9}{2}$ & z & $\frac{9}{2}$ & $\frac{9}{2}$ & $\frac{1}{2}$ & z & $\frac{0}{2}$ & $\frac{9}{2}$ & $\stackrel{\check{\nu}}{\rightleftharpoons}$ & $\stackrel{0}{2}$ & $\frac{9}{2}$ & $\stackrel{\breve{u}}{\check{0}}$ & z & $\frac{1}{2}$ & 2 & $\frac{1}{2}$ & $\frac{0}{2}$ & ' & $\frac{9}{2}$ & $\infty$ \\
\hline 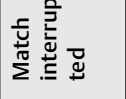 & i & i & z & i & in & i & $\stackrel{\breve{u}}{\succ}$ & $\stackrel{0}{z}$ & i & i & z & i & i & 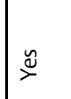 & $\stackrel{\check{\nu}}{\rightleftharpoons}$ & $\stackrel{0}{z}$ & ì & $\stackrel{0}{2}$ & I & in & $\tilde{\infty}$ \\
\hline 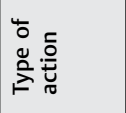 & 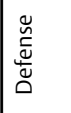 & 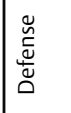 & 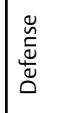 & 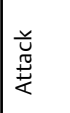 & 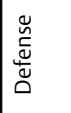 & 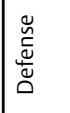 & 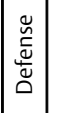 & $\begin{array}{l}\text { 总 } \\
\text { 菨 }\end{array}$ & 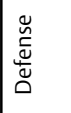 & 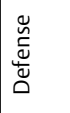 & 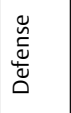 & 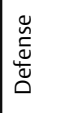 & 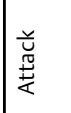 & 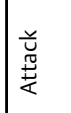 & 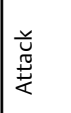 & $\begin{array}{l}\text { 总 } \\
\text { 莣 }\end{array}$ & 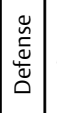 & 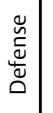 & & 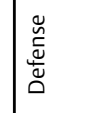 & 6 \\
\hline 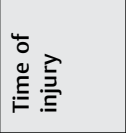 & 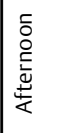 & 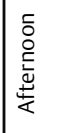 & 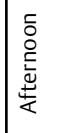 & 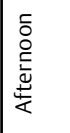 & 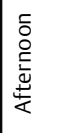 & 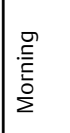 & 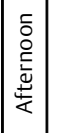 & 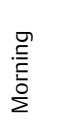 & 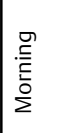 & 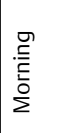 & 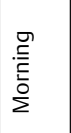 & 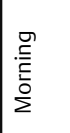 & 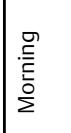 & 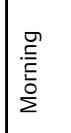 & 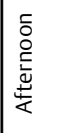 & 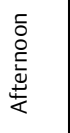 & 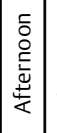 & 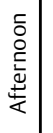 & I & 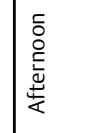 & 兄 \\
\hline 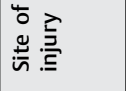 & 氡。 & 氡 & 离 & 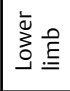 & 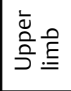 & 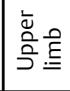 & \begin{tabular}{|l|}
$\square$ \\
$\widetilde{\Xi}$ \\
$\Phi$ \\
\end{tabular} & 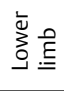 & 氡 & 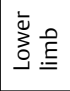 & 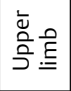 & 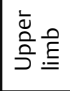 & 氡 & 离 & 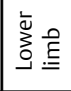 & 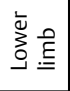 & \begin{tabular}{|l|}
$\square$ \\
$\tilde{\Xi}$ \\
$\Phi$ \\
\end{tabular} & 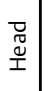 & I & 离 & $\overline{6}$ \\
\hline 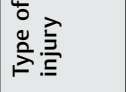 & 䓂 & 总 & 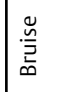 & 竒 & 总 & 总 & 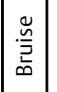 & 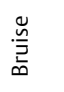 & 䓂 & 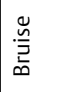 & 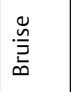 & 㕸 & 总 & 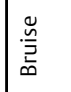 & 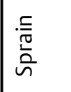 & 惢 & 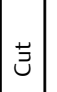 & 总 & , & 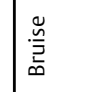 & $\infty$ \\
\hline
\end{tabular}

$+\frac{n}{0}$
$\dot{0}$
$\dot{2}$

\begin{tabular}{|c|c|c|c|c|c|c|c|c|c|c|c|c|c|c|c|c|c|c|c|c|c|}
\hline ฮ & $\sim$ & $m$ & $\sim$ & - & $\sim$ & $m$ & - & - & $\sim$ & $\sim$ & $\sim$ & $m$ & $m$ & $\sim$ & $\sim$ & - & & $r$ & & & $\sim$ \\
\hline पू⿹ & 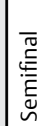 & 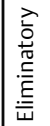 & 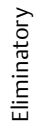 & 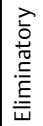 & 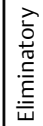 & 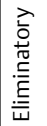 & & 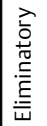 & 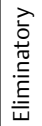 & $\begin{array}{l}\mid \overline{0} \\
0 \\
\frac{0}{E}\end{array}$ & 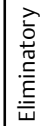 & & & & & & & & & & 产 \\
\hline
\end{tabular}

蒫

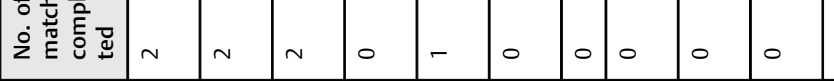

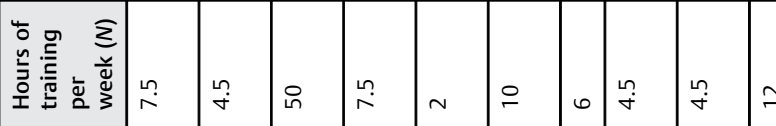

줗을

此

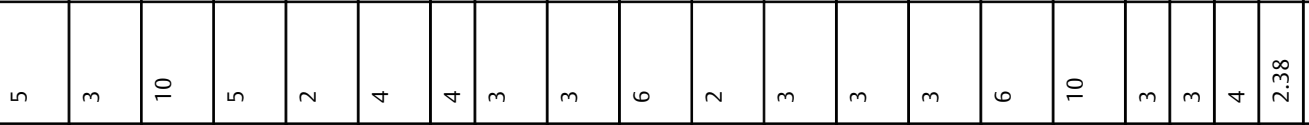

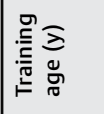

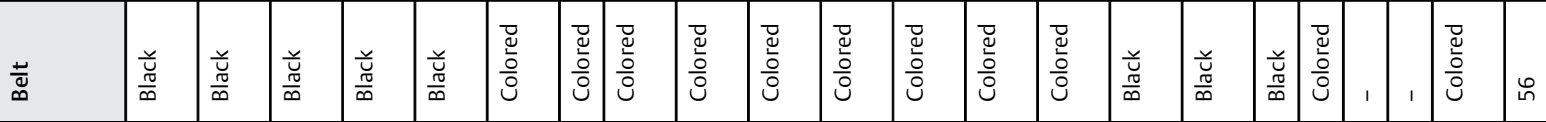

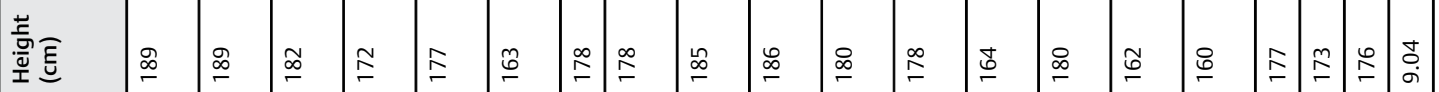

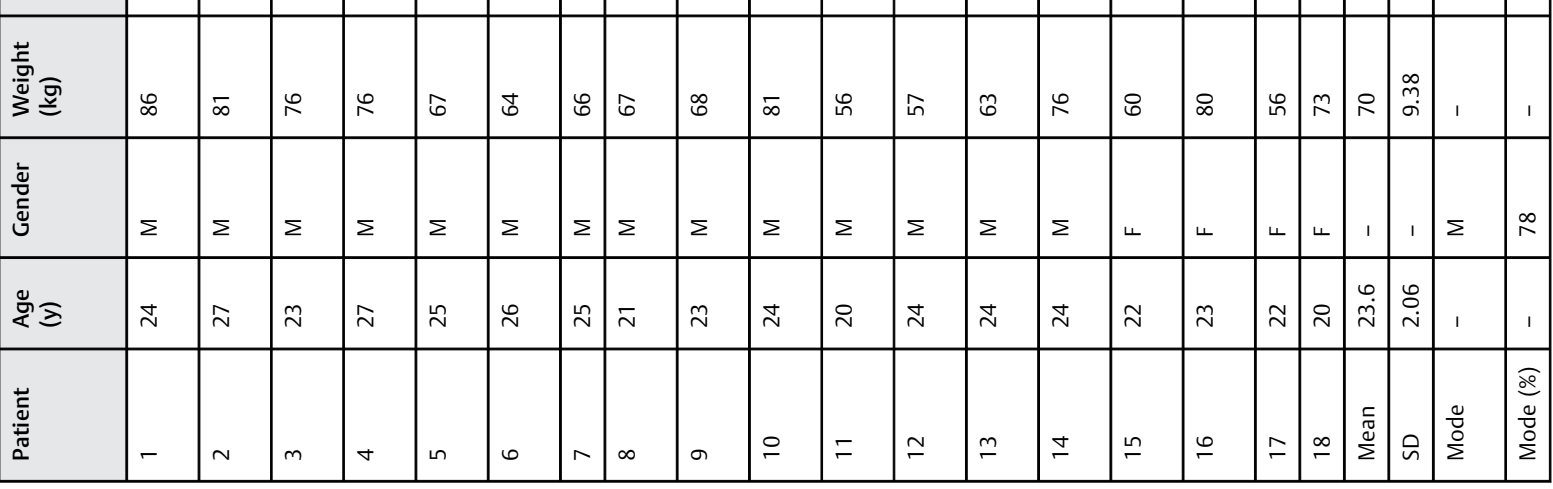

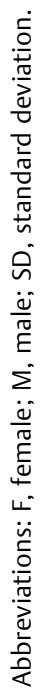


Table 2 Correlation analyses with Pearson's coefficients (“) and p values ( ${ }^{*}$ )

\begin{tabular}{|c|c|c|c|c|c|c|c|c|}
\hline Correlation ( $r$ ) & Age & Weight & Height & $\begin{array}{l}\text { Training } \\
\text { age }\end{array}$ & $\begin{array}{l}\text { No. of } \\
\text { weekly } \\
\text { training } \\
\text { sessions }\end{array}$ & $\begin{array}{l}\text { No. } \\
\text { of hours } \\
\text { of training } \\
\text { per week }\end{array}$ & $\begin{array}{l}\text { No. of } \\
\text { matches } \\
\text { completed }\end{array}$ & $\begin{array}{l}\text { No. of } \\
\text { rounds }\end{array}$ \\
\hline \multicolumn{9}{|l|}{ Age } \\
\hline Weight & $\begin{array}{l}-0.352^{\prime \prime} \\
0.152^{*}\end{array}$ & & & & & & & \\
\hline Height & $\begin{array}{l}-0.073^{\prime \prime} \\
0.773^{*}\end{array}$ & $\begin{array}{l}0.347^{\prime \prime} \\
0.158^{*}\end{array}$ & & & & & & \\
\hline Training age & $\begin{array}{l}0.265^{\prime \prime} \\
0.287^{*}\end{array}$ & $\begin{array}{l}-0.398^{\prime \prime} \\
0.101^{*}\end{array}$ & $\begin{array}{l}0.113^{\prime \prime} \\
0.654^{*}\end{array}$ & & & & & \\
\hline $\begin{array}{l}\text { No. of weekly training } \\
\text { sessions }\end{array}$ & $\begin{array}{l}-0.032^{\prime \prime} \\
0.900^{*} \\
\end{array}$ & $\begin{array}{l}0.463^{\prime \prime} \\
0.053^{*}\end{array}$ & $\begin{array}{l}-0.237^{\prime \prime} \\
0.343^{*} \\
\end{array}$ & $\begin{array}{l}-0.617^{\prime \prime} \\
0.006^{*} \\
\end{array}$ & & & & \\
\hline $\begin{array}{l}\text { No. of hours of training } \\
\text { per week }\end{array}$ & $\begin{array}{l}0.060^{\prime \prime} \\
0.814^{*}\end{array}$ & $\begin{array}{l}0.287^{\prime \prime} \\
0.249^{*}\end{array}$ & $\begin{array}{l}-0.186^{\prime \prime} \\
0.460^{*}\end{array}$ & $\begin{array}{l}-0.554^{\prime \prime} \\
0.017^{*}\end{array}$ & $\begin{array}{l}0.921^{\prime \prime} \\
< \\
0.0001^{*}\end{array}$ & & & \\
\hline $\begin{array}{l}\text { No. of matches } \\
\text { completed }\end{array}$ & $\begin{array}{l}-0.238^{\prime \prime} \\
0.341^{*}\end{array}$ & $\begin{array}{l}0.449 " \\
0.062^{*}\end{array}$ & $\begin{array}{l}0.405^{\prime \prime} \\
0.096^{*}\end{array}$ & $\begin{array}{l}-0.397^{\prime \prime} \\
0.103^{*}\end{array}$ & $\begin{array}{l}0.295^{\prime \prime} \\
0.235^{*}\end{array}$ & $\begin{array}{l}0.384^{\prime \prime} \\
0.116^{*}\end{array}$ & & \\
\hline No. of rounds & $\begin{array}{l}-0.208^{\prime \prime} \\
0.408^{*}\end{array}$ & $\begin{array}{l}-0.219^{\prime \prime} \\
0.382^{*}\end{array}$ & $\begin{array}{l}0.057^{\prime \prime} \\
0.822^{*}\end{array}$ & $\begin{array}{l}0.328^{\prime \prime} \\
0.184^{*}\end{array}$ & $\begin{array}{l}-0.325^{\prime \prime} \\
0.188^{*}\end{array}$ & $\begin{array}{l}-0.155^{\prime \prime} \\
0.539^{*}\end{array}$ & $\begin{array}{l}0.219^{\prime \prime} \\
0.383^{*}\end{array}$ & \\
\hline
\end{tabular}

requires both a high degree of physical coordination together with muscular and joint flexibility, qualities which are easier to develop if started at an early age. These athletes that started their practice later in life had called the medical staff on-site more than who started when was younger (at a mean of 19 years vs. 14 years of the other group).

A high standard deviation is also found for the number of hours of weekly training sessions (11.90); this shows that among these athletes there is a vast difference in the length of the training sessions. These data seem to be well above those to be found in the literature. ${ }^{6}$ Our correlation analysis showed that the more is the training age and the weekly hours of training, the more are the numbers of matches completed, even if injured. Beginners with a lowlevel belt suffered from injury more than experienced sub- jects as for other sports. Most of the subjects were males (78\%), probably due to the stronger kicks and punches used to hit the mark compared with females. Males showed a higher rate of injury with a substantially slight difference with females: 15 versus $11.8 \%$. In literature, there is a very little discrepancy regarding this topic. ${ }^{5,10}$ It seems that injured athletes have a low-level belt or are beginners, even if this point is still debated. ${ }^{1,11}$ The higher probability of injury was in the second round (56\%) when athletes tend to show their best technical and tactical capabilities after the first round of competitor "study." In the study by Ziaee et al, the highest number of injuries occurred in the third round, probably due to the fact that the competitor enters the last round with both mental and physical fatigue. ${ }^{3}$ Instead, in our sample, a high number of injuries occurred during the

Table 3 Results of Student's t-tests

\begin{tabular}{|c|c|c|c|c|c|c|c|}
\hline Continuous variables & Gender & Belt & $\begin{array}{l}\text { Time of } \\
\text { injury }\end{array}$ & $\begin{array}{l}\text { Type of } \\
\text { action }\end{array}$ & $\begin{array}{l}\text { Match } \\
\text { interrupted }\end{array}$ & $\begin{array}{l}\text { Competitor } \\
\text { penalized }\end{array}$ & $\begin{array}{l}\text { Medical team } \\
\text { intervention }\end{array}$ \\
\hline Age & 0.02 & Ns & Ns & Ns & Ns & Ns & Ns \\
\hline Weight & Ns & Ns & Ns & Ns & Ns & Ns & Ns \\
\hline Height & 0.03 & Ns & Ns & 0.016 & Ns & ns & Ns \\
\hline Training age & Ns & 0.0009 & 0.006 & Ns & Ns & 0.011 & 0.046 \\
\hline $\begin{array}{l}\text { No. of weekly training } \\
\text { sessions }\end{array}$ & Ns & Ns & Ns & Ns & Ns & Ns & Ns \\
\hline $\begin{array}{l}\text { No. of hours of training } \\
\text { per week }\end{array}$ & Ns & Ns & Ns & Ns & Ns & Ns & Ns \\
\hline $\begin{array}{l}\text { No. of matches } \\
\text { completed }\end{array}$ & Ns & 0.02 & 0.02 & Ns & Ns & 0.027 & Ns \\
\hline No. of rounds & $\mathrm{Ns}$ & Ns & Ns & Ns & $\mathrm{Ns}$ & Ns & $\mathrm{Ns}$ \\
\hline
\end{tabular}

Abbreviation: Ns, not significant. 
elimination rounds (78\%) and the first match of the day (72\%), indicating that it is not the fatigue that plays a fundamental role in the cause of injuries. Pieter also adds that during the first match a wider range of technical abilities is practiced and that the athletes are less inclined to present an injury when the competition is drawing to a close. ${ }^{4}$ As previously reported by other authors, ${ }^{6,12}$ in our survey bruising is the most common injury (89\%), particularly in the lower limbs (61\%). On the other hand, Ziaee et al found injuries in the upper limbs the most frequent, due to the fact that Iranian athletes mostly use the upper limbs during the fight with respect to other countries. ${ }^{3}$ Similarly, Pieter reported that head and neck were more prone to injury probably due to a different fighting style. ${ }^{13}$ In our study most of the athletes were able to finish the match (83\%) and did not call the medical team on-site (78\%): this means that most injuries were of low severity. ${ }^{14,15}$

The analysis of penalization of the opponent following an injury has shown that the action causing the injury was nearly always correct (89\%), that means that athletes usually follow the discipline and for the scope of injury prevention there is no need to revise the punitive actions. A high correlation between height and the number of matches completed was found in the group of black belt athletes and in one of the athletes that finished the game even if injured. Being tall in Taekwondo is usually an advantage because this allows the athlete to reach the opponent more easily and at the same time to keep a good safe distance from the opponent, reducing the entity of the hits taken: this is the reason why smaller athletes get mostly injured while attacking.

There are several limitations in this study. A scientifically validated questionnaire was not used, but we had to formulate one ex novo to include particular aspects of Taekwondo that did not appear in any other schedules. The number of athletes examined in this study was small even if a national competition was evaluated: the statistical power is, therefore, to be carefully interpreted.

However, this survey found some brand new risk factors for injury as starting to practice in late age, weekly training sessions with a few number of hours, male sex, low-level belt, elimination rounds, the first match of the day, second round, and height.
A study with a higher sample size and a specific questionnaire for injuries related to Taekwondo may be useful in future to understand the type better and frequency of lesions in this sport.

\section{References}

1 Lystad RP, Swain MS, Graham PL. Risk factors for injury in Olympic-style competition taekwondo: a systematic review. J Sports Med Phys Fitness 2013;53(06):655-664

2 Altarriba-Bartes A, Drobnic F, Til L, Malliaropoulos N, Montoro JB, Irurtia A. Epidemiology of injuries in elite taekwondo athletes: two Olympic periods cross-sectional retrospective study. BMJ Open 2014;4(02):e004605

3 Ziaee V, Rahmani S-H, Rostami M. Injury rates in Iranian taekwondo athletes; a prospective study. Asian J Sports Med 2010; 1(01):23-28

4 Pieter W. Martial arts injuries. Med Sport Sci 2005;48:59-73

5 Kazemi M, Chudolinski A, Turgeon M, Simon A, Ho E, Coombe L. Nine year longitudinal retrospective study of Taekwondo injuries. J Can Chiropr Assoc 2009;53(04):272-281

6 Kazemi M, Shearer H, Choung YS. Pre-competition habits and injuries in Taekwondo athletes. BMC Musculoskelet Disord 2005; 6:26-34

7 Pieter W, Fife GP, O'Sullivan DM. Competition injuries in taekwondo: a literature review and suggestions for prevention and surveillance. Br J Sports Med 2012;46(07):485-491

8 Zemper ED, Pieter W. Injury rates during the 1988 US Olympic Team Trials for taekwondo. Br J Sports Med 1989;23(03):161-164

9 Pieter W, Zemper ED. Injury rates in children participating in taekwondo competition. J Trauma 1997;43(01):89-95, discussion 95-96

10 Lystad RP, Graham PL, Poulos RG. Exposure-adjusted incidence rates and severity of competition injuries in Australian amateur taekwondo athletes: a 2-year prospective study. $\mathrm{Br} J$ Sports Med 2013;47(07):441-446

11 Schlüter-Brust K, Leistenschneider P, Dargel J, Springorum HP, Eysel P, Michael JW-P. Acute injuries in Taekwondo. Int J Sports Med 2011;32(08):629-634

12 Yard EE, Knox CL, Smith GA, Comstock RD. Pediatric martial arts injuries presenting to Emergency Departments, United States 1990-2003. J Sci Med Sport 2007;10(04):219-226

13 Pieter W, Rostami M, Ziaee V. An addendum to injury rates in Iranian taekwondo athletes; a prospective study. Asian J Sports Med 2010;1(02):117-121

14 Kazemi M. Relationships between injury and success in elite Taekwondo athletes. J Sports Sci 2012;30(03):277-283

15 Lystad RP, Pollard H, Graham PL. Epidemiology of injuries in competition taekwondo: a meta-analysis of observational studies. J Sci Med Sport 2009;12(06):614-621 